Journal of

Accident and

Emergency

Medicine 1994

11, 178-182

\title{
Evaluation of topical ibuprofen cream in the treatment of acute ankle sprains
}

\author{
J.CAMPBELL \& T.DUNN
}

Accident and Emergency Department, St John's Hospital at Howden, Livingston

\section{SUMMARY}

One hundred patients who presented to the accident and emergency (A\&E) department with an acute ankle sprain were entered into a study to determine the efficacy of topical ibuprofen cream by using a double-blind placebo controlled design in a single type of soft-tissue injury. The subjects were given either topical ibuprofen cream or a placebo cream in addition to the standard management of the department. Patients kept diaries recording walking ability and pain visual analogue scales for resting, standing and walking. A total of 51 patients returned diaries that were suitable for analysis. Patients using the topical ibuprofen cream had significant reduction in pain scores over the first $48 \mathrm{~h}$ of treatment.

Key words: ankle sprain, ibuprofen, topical

\section{INTRODUCTION}

Non-steroidal anti-inflammatory drugs (NSAIDs) are known to have a beneficial therapeutic effect for acute soft-tissue injuries, ${ }^{1}$ but systemic administration can be associated with side-effects. Many topical preparations of NSAIDs have become available in recent years with the promise of efficacy coupled with improved tolerableness. The purpose of this study was to evaluate the efficacy of topical ibuprofen in a single injury type (acute ankle sprain) using a double-blind, placebo controlled design. The chosen outcome measures were relief of symptoms and return to normai function. Although these are subjective they are also most relevant clinically.

\section{SUBJECTS AND METHODS}

The study was conducted in the A\&E department of St John's Hospital in Livingston, West Lothian. One hundred patients aged between 16 and 60 years who presented to the A\&E department with a soft- tissue ankle injury during the study period were invited to participate. Patients were included in the study if they presented within $24 \mathrm{~h}$ with a unilateral injury and no apparent fracture or instability. They were excluded if there was any pre-existing ankle problem or if they were already taking any nonsteroidal anti-inflammatory medication.

The A\&E doctor seeing the patient obtained informed consent from the patient and completed an initial assessment form. Radiographs were requested only if there was suspicion of bony injury, according to the department guidelines. ${ }^{2}$ Patients were given a numbered tube of cream containing either placebo cream (the identical cream base without the active ingredient) or ibuprofen cream the cream used in this study was Proflex (Zyma Holmwood, UK). This contains 5\% ibuprofen in a cream base. It is currently available with or without a prescription. The randomization of treatment was carried out by Zyma, and the tubes were dispensed from the hospital pharmacy who held a record of the codes. Subjects were given written instructions to apply the cream in a dose of four inches four times daily. They were also given a supply of paracetamol tablets to use if necessary as rescue medication. Subjects were asked to keep a daily diary of symptoms until they were recovered. They were given two diary booklets lasting 1 week each. They were asked to return to the A\&E department for review if they still had any symptoms after 2 weeks, or earlier if concerned. The diaries contained simple tick boxes asking about function, use of ice, tubigrip, and paracetamol, and also three visual analogue scales measuring their pain at rest, standing and walking. Reply-paid envelopes were provided for returning each week's diary. The diaries contained a page of specific advice on self-management of their injury - rest and regular icing for 48 hours, and then walking encouraged with regular exercises to be performed. 
Topical ibuprofen cream and ankle sprain

\section{RESULTS}

One hundred patients were entered into the trial and randomized to one or other treatment group, with 51 treated with Proflex, and 49 with placebo. Six subjects were excluded (three presented more than $24 \mathrm{~h}$ after injury, two had missing forms, and one appeared twice). Fifty-five subjects returned diaries $(55 \%)$ including four of the excluded subjects, leaving 51 subjects suitable for analysis (26 on Proflex and 25 on placebo). Table 1 shows the characteristics of the groups at randomization, and those eligible for analysis. At both stages the groups appear to be homogeneous.

The means of the three visual analogue scales, and of the total of all three, are shown in Fig. 1. The Bonferroni adjustment to $t$-tests was used for seven comparisons between the two groups. The difference between the two groups is significant $(P<0.05)$ over the first $48 \mathrm{~h}$ of treatment (i.e. days 2 and 3 ), and the significance is maintained when the figures are adjusted for the difference in initial pain scores by using day 1 values as a covariate (Table 2).

Patients recorded their walking ability on a four point scale ('I can walk and run comfortably', 'walking is comfortable except on uneven ground', 'I am comfortable standing, but walking is uncomfortable', 'taking weight on my ankle is uncomfortable'). Figure 2 shows improvement in walking ability over the first 7 days. Although there is possibly a more rapid improvement in function in the Proflex goup the difference is not significant using the Chi-squared test.

There was no significant difference in the use of rescue medication (paracetamol). Subjects were asked to apply the cream four times daily until recovered. Figure 3 shows the frequency of cream application, with a fairly rapid fall off in compliance. There was no difference in frequency of application between the two groups.

Patients' comments were recorded. The only side-

Table 1. Characteristics of patients entered into trial $(n=100)$, and of those eligible for analysis $(n=51)$

\begin{tabular}{|c|c|c|c|c|c|}
\hline & & \multicolumn{2}{|c|}{ Patients entered } & \multicolumn{2}{|c|}{ Patients analysed } \\
\hline & & Proflex & Placebo & Proflex & Placebo \\
\hline \multirow{2}{*}{ Sex } & Male & 36 & 28 & 17 & 16 \\
\hline & Female & 15 & 21 & 9 & 9 \\
\hline \multirow[t]{2}{*}{ Age } & Mean & 29.4 & 29.3 & 26.4 & 34.3 \\
\hline & std err & 1.7 & 2 & 2.1 & 3 \\
\hline \multirow[t]{4}{*}{ Occupation } & Sedentary & 13 & 13 & 8 & 8 \\
\hline & Standing-light & 18 & 14 & 10 & 8 \\
\hline & Standing-heavy & 15 & 16 & 7 & 7 \\
\hline & Home/unemployed & 4 & 5 & 1 & 2 \\
\hline Time interval between & Mean (hours) & 9.2 & 8.8 & 7.9 & 8.3 \\
\hline injury and presentation & std err & 1.2 & 1.3 & 1.3 & 1.5 \\
\hline \multirow[t]{2}{*}{ Bruising } & Present & 28 & 31 & 14 & 17 \\
\hline & Absent & 22 & 18 & 12 & 8 \\
\hline \multirow[t]{3}{*}{ Ankle movement } & Full range & 17 & 15 & 11 & 8 \\
\hline & Slight restriction & 27 & 28 & 13 & 13 \\
\hline & Marked restriction & 6 & 6 & 2 & 4 \\
\hline \multirow[t]{4}{*}{ Ability to weight bear } & Walking normally & 0 & 0 & 0 & 0 \\
\hline & Slight limp & 24 & 26 & 13 & 14 \\
\hline & Limping badly & 21 & 17 & 12 & 9 \\
\hline & Unable to weight bear & 5 . & 6 & 1 & 2 \\
\hline \multirow[t]{4}{*}{ Swelling } & None & 0 & 1 & 0 & 1 \\
\hline & Mild & 23 & 22 & 14 & 9 \\
\hline & Moderate & 22 & 19 & 11 & 13 \\
\hline & Severe & 5 & 7 & 1 & 2 \\
\hline \multirow[t]{2}{*}{ Radiograph } & Yes & 38 & 30 & 21 & 14 \\
\hline & No & 12 & 19 & 5 & 11 \\
\hline \multirow{3}{*}{$\begin{array}{l}\text { Mean pain V.A.S. } \\
\text { on presentation }\end{array}$} & Resting & 35.9 & 42.5 & 33.9 & 42.3 \\
\hline & Standing & 61.5 & 65 & 60.7 & 65.8 \\
\hline & Walking & 80.4 & 80.7 & 80 & 81.3 \\
\hline
\end{tabular}




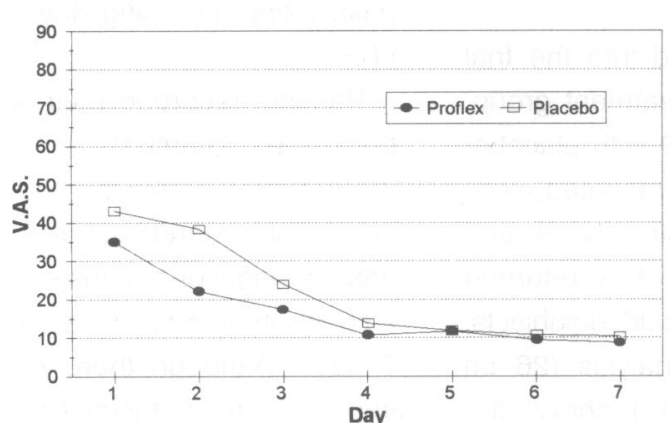

Pain on walking

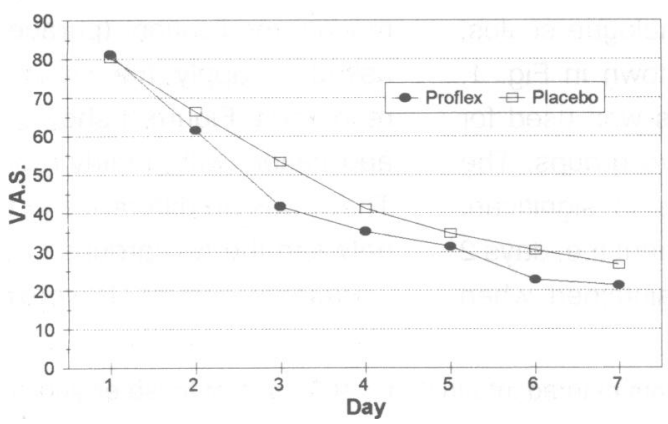

Fig. 1. Pain visual analogue scores over first week.

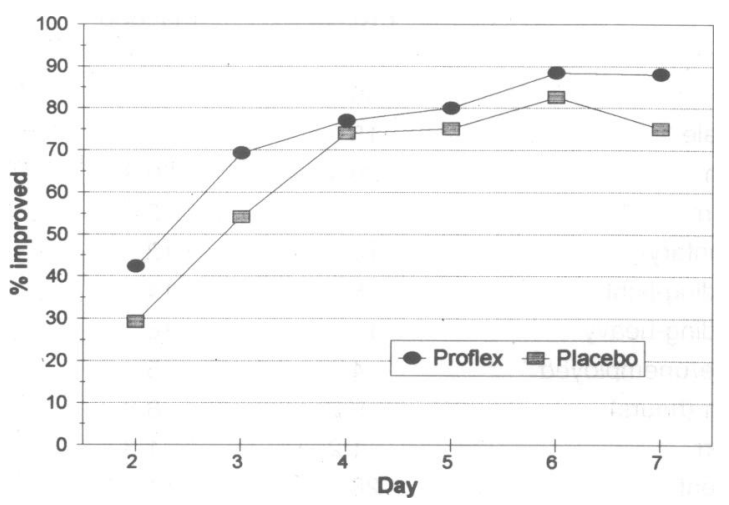

Fig. 2. Improvement in walking ability.

Table 2. Significance of mean total V.A.S. pain scores

\begin{tabular}{llrrrrrrr}
\hline & Day & 1 & 2 & 3 & 4 & 5 & 6 & 7 \\
\hline Total mean & Placebo & 188.5 & 157.7 & 116.7 & 82.2 & 68.8 & 59.3 & 52.8 \\
V.A.S. scores & Proflex & 178.6 & 129.3 & 87.9 & 65.2 & 60.2 & 45.7 & 41.8 \\
& difference & 9.9 & 28.4 & 28.8 & 17 & 8.6 & 13.6 & 11 \\
& $\mathrm{t}$ & 1.4 & 3.9 & 4 & 2.4 & 1.2 & 1.9 & 1.5 \\
Adjusted & Placebo & 185.5 & 154.7 & 113.7 & 79.2 & 65.9 & 56.3 & 49.8 \\
scores for day & Proflex & 181.2 & 131.9 & 90.5 & 67.8 & 62.8 & 48.3 & 44.3 \\
one difference & difference & 4.3 & 22.8 & 23.2 & 11.4 & 3.1 & 8 & 5.5 \\
& $\mathrm{t}$ & 0.6 & 3.2 & 3.2 & 1.6 & 0.4 & 1.1 & 0.8 \\
\hline
\end{tabular}

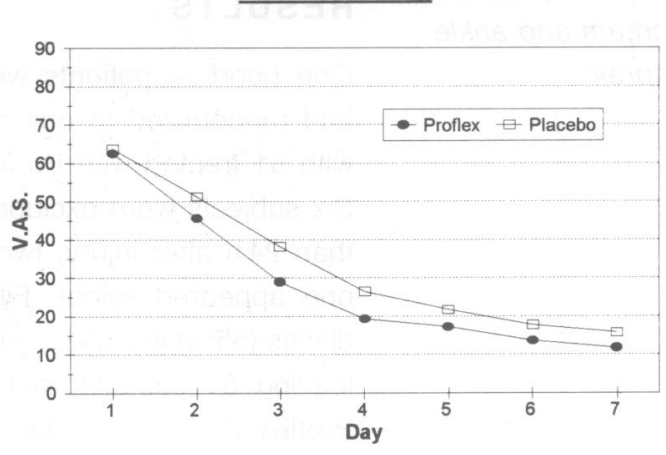

Total V.A.S.scores

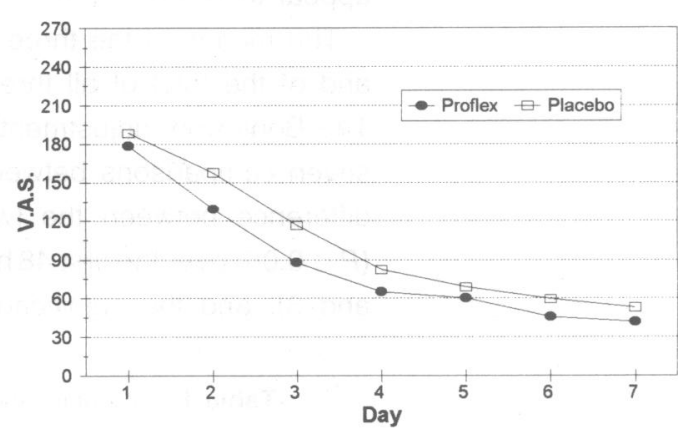

effect classified as possibly related to Proflex was a headache in one patient. Only 25 of the 51 subjects returned a diary for the second week. There were no significant differences between the two groups in week 2 on any of the measures.

\section{DISCUSSION}

Management of soft tissue injuries is often suboptimal. ${ }^{3}$ Ankle sprains are said to account for $33 \%$ of sports injuries, and can have serious sequelae. ${ }^{4}$ Anti-inflammatory medication, both oral and topical, has been advocated for the treatment of soft-tissue 
Topical ibuprofen cream and ankle sprain

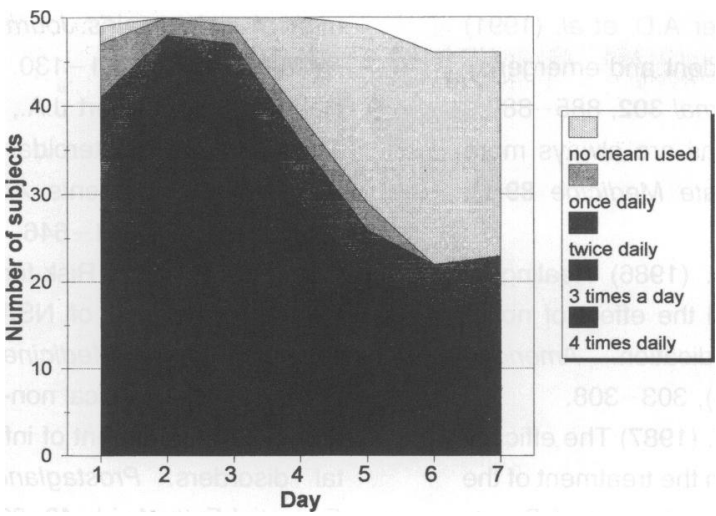

Fig. 3. Frequency of cream application.

injuries. They are effective analgesics and can therefore help early return of function. Evidence for the efficacy of NSAIDs in this situation is mixed. In a review of 35 papers on the clinical effects of NSAIDs ${ }^{1}$ only 15 used a placebo group, of which eight studied a single injury type. Four of these eight studies showed a significant benefit from treatment.

In some studies various objective measures such as degree of swelling, range of movement, degree of tenderness have been measured. The view was taken that these measures were often of doubtful clinical relevance, and that although functional impairment and degree of pain were subjective they represented the goals of treatment.

One study of oral ibuprofen in the treatment of 61 acute ankle sprains showed no significant benefit over placebo. ${ }^{6}$ Evaluation was carried out on day 1 , 4 and 8 . Thus any difference over the first 3 days would have gone unrecorded. However, there was a consistent trend for all outcome measures in favour of ibuprofen over placebo.

A double-blind placebo controlled study of ibuprofen cream in 80 patients with sprains and contusions found a significant difference in physicians' assessment of speed of resolution, relief of pain and swelling, and return of function. ${ }^{7}$ However, most patients improved rapidly and there was no difference in patients' rating of symptoms. Another study comparing oral diclofenac potassium with ibuprofen and placebo in ankle injuries showed a significant reduction in swelling, tenderness, and pain on passive movement by day $3 .{ }^{8}$

The healing process of muscle strains may be delayed by the use of nonsteroidal anti-inflammatory medication. ${ }^{5}$ Histological examination of an animal model showed a diminished inflammatory response with a delay in regaining muscle tensile strength. ligaments rather than muscles. Another animal model study on ligament healing showed that administration of NSAID (piroxicam) resulted in increased tensile strength at day 14 , but no difference by day 21.9

Users of oral NSAIDs have a three-fold increase in risk for developing a serious adverse gastrointestinal (GI) event. ${ }^{10}$ Oral NSAIDs can have systemic side-effects, such as gastritis and GI haemorrhage, hypersensitivity, headache, dizziness, blood disorders and fluid retention. In severe chronic rheumatic conditions the benefits may outweigh the risks. However, for acute, localized inflammatory disorders of soft tissues the risks may not be justified. Topical therapy represents an attractive alternative. ${ }^{11}$

Application of ibuprofen cream to the skin produces plasma levels which are about $5 \%$ of those that follow a standard oral dose of ibuprofen, ${ }^{12}$ although local muscle and fascia levels may be 500 times higher. ${ }^{13}$ Ibuprofen is absorbed almost as rapidly after topical as oral administration, ${ }^{12}$ although other topical preparations are much slower. ${ }^{11}$ Topical ibuprofen has a longer half-life than the oral preparation ( $8-12 \mathrm{~h}$ compared with $2 \mathrm{~h}$ ). Studies show that the drug is present in synovial fluid, muscle and capsular tissue after topical absorption. ${ }^{13}$

This study shows that the use of topical ibuprofen is associated with a statistically significant reduction in pain over the first $\mathbf{4 8}$ hours of treatment following an acute ankle sprain. The amount of pain reduction is of the order of $10 \%$ more with ibuprofen cream than with placebo. The application of any cream may have beneficial effects by massaging the swollen tissues. The extent of this effect, if any, is not known and would have affected both groups equally. Although oral NSAID therapy is likely to confer a similar benefit this is at the risk of potentially serious side-effects.

\section{ACKNOWLEDGEMENTS}

We are grateful to $\mathrm{Dr}$ Freeland, Consultant in Accident \& Emergency, and our other colleagues in the A\&E department for their help and support.

\section{REFERENCES}

1. Almekinders L.C. (1990) The efficacy of nonsteroidal anti-inflammatory drugs in the treatment of ligament injuries. Sports Medicine 9(3), 137-142.

2. Dunlop M.G., Beattie T.F., White G.K. et al. (1986) Guidelines for selective radiological assessment of inversion ankle injuries. British Medical Journal 293 603-605. 


\section{J. Campbell \& \\ T. Dunn}

3. Packer G.J., Goring C.C., Gayner A.D. et al. (1991) Audit of ankle injuries in an accident and emergency department. British Medical Journal 302, 885-887.

4. Stanley K.L. (1991) Ankle sprains are always more than 'just a sprain'. Postgraduate Medicine 89(1), 251-255.

5. Almekinders L.C. \& Gilbert J.A. (1986) Healing of experimental muscle strains and the effect of nonsteroidal anti-inflammatory medication. American Journal of Sports Medicine 14(4), 303-308.

6. Dupont M., Beliveau, Theriault G. (1987) The efficacy of anti-inflammatory medication in the treatment of the acutely sprained ankle. American Journal of Sports Medicine 15(1), 41-45.

7. Ramesh N. \& Steuber U. (1983) Results of a doubleblind study of Dolgit ${ }^{\circledR}$ cream in accident and sports related injuries in medical practice. Therapie Woche 33, 4563-4570.

8. Moran M. (1991) Double-blind comparisons of diclofenac potassium, ibuprofen, and placebo in the treat- ment of ankle sprains. Journal of International Medical Research 19(2), 121-130.

9. Dahners L.E., Gilbert J.A., Lester G.E. et al. (1988) The effect of a nonsteroidal anti-inflammatory drug on the healing of ligaments. American Journal of Sports Medicine 16(6), 641-646.

10. Gabriel S.E. (1991) Risk for serious GI complications related to the use of NSAIDs - a meta-analysis. Annals of Internal Medicine 115(10), 787-796.

11. White S. (1991) Topical non-steroidal anti-inflammatory drugs in the treatment of inflammatory musculoskeletal disorders. Prostaglandins, Leukotrienes and Essential Fatty Acids 43, 209-222.

12. Mondino A., Zanolo G., Giachetti C. et al. (1983) Humankinetische untersuchungen mit ibuprofen. Med Welt 34, 1052-1054.

13. Peters H., Chlud K., Berner G. et al. (1987) Zur perkutanen kinetik von ibuprofen. Aktuelle Rheumatologie 12, 208-211. 